\title{
Caracterización de una infusión de cascarilla de cacao
(Theobroma cacao L., var. Arriba) con hierbas aromáticas
}

\author{
Characterization of a cocoa (Theobroma cacao L., var. Arriba) \\ husks infusion with aromatic herbs
}

\author{
Teneda Llerena, W.F. ${ }^{a *}$, Ah-Hen, K. ${ }^{b}$, Lemus-Mondaca, R. ${ }^{c}$ \\ ${ }^{a}$ Facultad de Ciencias Administrativas, Universidad Técnica de Ambato, Ambato, Ecuador. \\ ${ }^{b}$ Instituto de Ciencia y Tecnología de Alimentos, Facultad de Ciencias Agrarias, Universidad Austral de Chile, Valdivia, Chile. \\ ${ }^{c}$ Departamento Ciencia de los Alimentos y Tecnología Química, Facultad de Ciencias Químicas y Farmacéuticas, \\ Universidad de Chile, Santiago, Chile.
}

\begin{tabular}{l} 
A R T I C L E I N F O \\
\hline Article history: \\
Received 24.05.2018 \\
Accepted 24.09.2018 \\
\hline Keywords: \\
Husks of cocoa, \\
Infusion, \\
Guayusa, \\
Peppermint, \\
Lemongrass \\
\hline Original Research Article, \\
Food Processing and Quality \\
\hline *Corresponding author: \\
William Teneda \\
E-mail address: \\
wf.teneda@uta.edu.ec
\end{tabular}

\begin{abstract}
A B S T R A C T
In this study an assessment through sensory analysis and physico-chemical characterization of prepared infusions made of the cocoa husks, discarded during the roasting process to obtain cocoa paste, mixed with aromatic herbs was performed. Infusion of the husks from Cocoa Arriba (Theobroma cacao L.) was prepared mixed with 3 different varieties of dried herbs, including guayusa (Ilex guayusa Loes), lemongrass (Cymbopogon citratus (DC.) Stapf) and peppermint (Mentha piperita L.) in varying proportions, that sought appropriate considerations of quality and safety specifications demanded by consumers. An experimental design with 2 factors at 3 levels was applied, with varying percentages of husks $\left(\mathrm{a}_{0}: 80 \%, \mathrm{a}_{1}: 70 \%, \mathrm{a}_{2}: 60 \%\right)$ and dried herbs $\left(\mathrm{b}_{0}:\right.$ lemongrass, $\mathrm{b}_{1}:$ guayusa, $b_{2}$ : peppermint), which accounted for a total of 9 treatments plus a control (100\% husks). The assays were performed in triplicates. The cocoa husks had $9.78 \pm 0.12 \%$ moisture, $5.37 \%$ lipids, a content of total polyphenolics of $2135 \pm 10 ; 468 \pm 10 ; 662 \pm 10$ and $977 \pm 10 \mathrm{mg}$ GAE (gallic acid equivalent) $100 \mathrm{~g}^{-1}$ sample in the cacao husk, lemongrass, guayusa and peppermint, respectively. A balanced incomplete block design was used for the sensory analysis and treatments were assessed by 18 semi-trained judges, implying five samples per judge, resulting into nine replicates for each treatment performed in duplicate. Sensory analysis included the evaluation of color, odor, flavor and acceptability, and the formulation best valued by the tasters was that containing $80 \%$ cocoa husks and $20 \%$ guayusa. This formulation had a moisture content of $9.42 \pm 0.18 \%$, a pH value of 6.64 ; soluble solids $0.4^{\circ}$ brix and acidity of $0.014 \%$ reported as citric acid, being microbiologically stable at $18^{\circ} \mathrm{C}$ with a shelf-life of 148 days.
\end{abstract}

\section{RESUMEN}

Se caracterizó mediante análisis fisicoquímico y sensorial la preparación de infusiones de cascarilla del cacao, eliminada en el proceso de tostado para obtención de pasta, en mezcla con hierbas aromáticas. La infusión de la cascarilla de Cacao Arriba (Theobroma cacao L.) se preparó con 3 distintas hierbas deshidratadas, incluyendo guayusa (Ilex guayusa Loes), hierba luisa o hierba limón (Cymbopogon citratus (DC.) Stapf) y menta (Mentha piperita L.), combinadas en diferentes porcentajes bajo especificaciones de calidad e inocuidad que cumplen con las exigencias de los consumidores. Se aplicó un diseño experimental de dos factores a tres niveles en triplicado, con un porcentaje variable de cascarilla de cacao ( $\left.a_{0}: 80 \%, a_{1}: 70 \%, a_{2}: 60 \%\right)$ y plantas deshidratadas $\left(\mathrm{b}_{0}\right.$ : hierba luisa, $\mathrm{b}_{1}$ : guayusa, $\mathrm{b}_{2}$ : menta), obteniendo un total de 9 tratamientos además del testigo (100\% cascarilla). La cascarilla de cacao variedad Arriba tuvo 9,78 \pm 0,12\% de humedad, 5,37\% de lípidos, y un contenido de polifenoles totales de $2135 \pm 10 ; 468 \pm 10 ; 662 \pm 10$ y $977 \pm 10 \mathrm{mg}$ AGE (ácido gálico equivalente) $100 \mathrm{~g}^{-1}$ en muestras de cascarilla de cacao, hierba luisa, guayusa y menta, respectivamente. Para la evaluación sensorial se utilizó un diseño de bloques incompletos equilibrados con los tratamientos evaluados por 18 jueces semi-entrenados, generándose cinco muestras por catador, de forma que hubo nueve réplicas para cada tratamiento realizado en duplicado. En el análisis sensorial (color, aroma, sabor y aceptabilidad), la formulación mejor valorada fue la infusión con $80 \%$ cascarilla de cacao y $20 \%$ de guayusa.

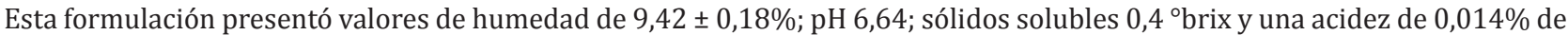
ácido cítrico, y es microbiológicamente estable a $18{ }^{\circ} \mathrm{C}$ con una vida útil de 148 días.

Palabras clave: Cascarilla de cacao, infusión, guayusa, menta, hierba Luisa. 


\section{INTRODUCCIÓN}

El cacao es de gran importancia en las actividades agrícolas del Ecuador, siendo la tercera mayor exportación que genera fuentes de trabajo directas e indirectas. Es parte esencial de la cotidianidad de muchas comunidades. Los granos de cacao comercializados hoy en día son semillas de un pequeño arbusto de la especie Theobroma cacao L., de la familia Esterculiácea. Es el único de su especie con un valor comercial y se subdivide principalmente en dos grupos, Criollo y Forastero. Un tercer grupo conocido bajo el nombre de Trinitario es básicamente un híbrido de los dos grupos anteriores.

El cacao ha sido cultivado en Ecuador desde la era incaica en el siglo 12 (INIAP, 1994). Al principio del siglo 20, Ecuador era el principal productor de cacao fino, siendo la variedad Arriba del grupo Criollo la más famosa (Lerceteau et al., 1997). Sólo el 5\% del cacao producido en el mundo pertenece a la categoría de cacao fino, de lo cual más del 65\% proviene del Ecuador, lo que lo convierte en el mayor productor de cacao fino en el mundo (Nestlé, 2017). La variedad nacional del Ecuador denominado Fino de Aroma y conocido también como el cacao "Arriba", cuya producción y exportación hace de Ecuador el líder mundial del cacao fino, posee una calidad única en el mundo debido a sus características aromáticas particulares, con aromas florales muy perceptivas (De la Mota, 2007).

La transformación del cacao significa básicamente la conversión en granos sin cáscara, licor, manteca, torta y polvo. La fabricación de chocolate incluye la mezcla y refinado del licor de cacao con la manteca de cacao y otros ingredientes, tales como la leche y el azúcar (Perego et al., 2004). El primer paso en el procesamiento del cacao en la planta industrial es la limpieza del grano, cuando se remueven las impurezas, para el descascarillado de la semilla, la cual es sometida a un proceso térmico dirigido. De esta manera, la humedad que pierde el grano ejerce una presión en la cascarilla, separándola del mismo. Luego, por vibración, esta cascarilla es desviada de la línea principal de producción, quedando como un producto de descarte (De la Mota, 2007). Sin embargo, la cascarilla de cacao, considerado desecho agroindustrial, contiene aún macro nutrientes (proteínas, carbohidratos, lípidos), micro nutrientes (vitaminas y minerales) de la fruta del cacao (Holland et al., 1991), como también polifenoles con actividades biológicas y antioxidantes (Arlorio et al., 2005), lo que presenta un gran potencial de uso como alimentos funcionales. Expertos en la fabricación de productos a base de cacao, determinan que el rendimiento de $100 \mathrm{~kg}$ de semillas de cacao es alrededor del $85 \%$, siendo el valor restante considerado como desechos. De estos desechos, sólo la cascarilla de cacao corresponde el $12 \%$ (Murillo, 2008). La cascarilla de cacao como todo alimento aporta nutricionalmente con macronutrientes (proteínas, carbohidratos, lípidos) y micronutrientes (vitaminas y minerales). Este desecho agro-industrial se considera como una fuente baja de energía debido a que presenta niveles de energía digestible menor a $2500 \mathrm{kcal} \mathrm{kg}^{-1}$, que es la base de la fibra para la nutrición animal (López, 2013).

Las plantas medicinales deben su acción a ciertos componentes denominados principios activos. Curiosamente, en muchos casos, estos principios activos son metabolitos secundarios de las plantas, es decir, sustancias aparentemente importantes para la planta y que en muchos casos se consideran como desechos metabólicos (Quer, 1964). Existen plantas con ingredientes funcionales que se han venido utilizando desde hace mucho tiempo y que son objeto de estudio en muchas partes del mundo por su utilidad en la prevención de enfermedades crónicas como el cáncer, el deterioro mental, visual y las enfermedades cardiovasculares entre otras (Kuriyama, 2008; Higdon y Frei, 2003). La demanda de productos con ingredientes funcionales en alimentos, hierbas o tés es creciente, especialmente en los países desarrollados como Estados Unidos, Japón, Canadá y algunos países de Unión Europea, que buscan oferta de buena calidad en los países en vías de desarrollo (Dávila et al., 2010).

La guayusa (Ilex guayusa Loes) contiene $2-3 \%$ de cafeína, lo cual es una cantidad superior a la que tiene el café o el té (Higdon y Frei, 2003). Este resultado corrobora definitivamente la cualidad fármaco dinámica atribuida a la planta por los pobladores de la región oriental. Ellos expresan que el agua de guayusa levanta las fuerzas, lo que equivale en términos médicos a que es un gran tónico y estimulante, cualidad que está relacionada científicamente con el alto contenido de cafeína (Dueñas et al., 2016).

La hierba luisa o hierba limón (Cymbopogon citratus (DC.) Stapf) perteneciente a la familia de las Gramíneas es una hierba conocida a nivel mundial por su aroma a limón y es consumido como infusión aromática. Las hojas frescas se utilizan también como aliño en la cocina tradicional. La infusión de las hojas de la hierba luisa (Cymbopogon citratus (DC.) Stapf) por su contenido de

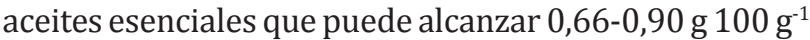
de hojas frescas, compuestos de aldehído citral, furfural, citronenal, mirceno, metil-heptenona, tripterpenos tales como cymbopogona y cymbopogonol, y alcoholes tales como berol y geraniol (Maiti et al., 2006), se usa como estimulante del sistema nervioso, las hojas mezclada con limón y raspadura se toma para combatir el frío y tratar el escalofrío, también ayuda a la digestión y es efectiva para tratar el dolor de cabeza, pecho, estómago, diarreas fuertes, gastritis, úlceras (De la Torre et al., 2008), se conoce también por sus propiedades anti-inflamatorias (Figueirinha et al., 2010; Blanco et al., 2009).

La menta, Mentha piperita L., es una planta herbácea con propiedades medicinales y pertenece a la familia de las Lamiaceae originaria del Mediterráneo 
(Desam et al., 2017). Las hojas de menta se usan en forma de infusión para la producción de jugo gástrico, favoreciendo las funciones digestivas del estómago. Tiene también propiedades antiinflamatorias, facilita las digestiones, elimina los gases y flatulencias, alivia la acidez estomacal, disminuye los dolores y convulsiones y combate los mareos y náuseas (Marcillo y Naranjo, 2012). Estas propiedades se deben al contenido de aceites esenciales como mentol $(36,02 \%)$, mentona $(24,56 \%)$, mentilacetato $(8,95 \%)$, mentofurano $(6,88)$ entre otros (Kapp et al., 2013). Contiene también carburos terpénicos, polifenoles y flavonoides derivados del apigenol y del luteolol, ácidos fenólicos y ácidos triterpénicos (Kapp et al., 2013).

La mayor dificultad que se presenta cuando se prepara la elaboración de nuevos productos o el aprovechamiento de subproductos industriales, tal como la cascarilla de cacao, es la falta de información sobre las cualidades nutricionales que poseen estos productos. Por lo general, la preocupación que existe por el reciclaje de supuestos residuos industriales ha impulsado la comunidad científica, especialmente en los trabajos a nivel industrial, en donde los procesos de transformación generan valiosos subproductos que albergan grandes potenciales de uso. El uso de la cascarilla del cacao en la industria de alimentos y bebidas funcionales, de suplementos alimenticios y nutricionales, aumenta consistentemente, según indican las cifras de mercado y la demanda creciente de los consumidores por alimentos saludables (Castillejo et al., 2006). Por lo cual, el presente trabajo tiene como objetivo evaluar el potencial uso de la cascarilla de cacao (Theobroma cacao L.) de la variedad Arriba a través de la caracterización sensorial y fisicoquímica de una infusión preparada a partir de la cascarilla sola y en combinación con varias hierbas secas, incluyendo guayusa (Ilex guayusa Loes), hierba luisa (Cymbopogon citratus (DC) Stapf) y menta (Mentha piperita L.) en varias proporciones.

\section{MATERIAL Y MÉTODOS}

\section{Materia Prima}

Se empleó cascarilla del cacao (Theobroma cacao L.) variedad Arriba proveniente de la Asociación de productores de Cacao APROCAI, ubicado en el Cantón Cumandá de la provincia de Chimborazo, Ecuador, cuya altitud varía de 300 hasta $1900 \mathrm{msnm}$, con temperatura promedio de $20^{\circ} \mathrm{C}$. Las hierbas fueron adquiridas en supermercados del comercio formal de la ciudad de Ambato, Ecuador.

\section{Diseño experimental}

Se aplicó un diseño experimental completamente aleatorio con arreglo factorial, A x B con dos réplicas, determinándose un número de nueve tratamientos más un control. Factor A: Porcentaje de cascarilla. $\mathrm{a}_{0}$ : $80 \%, a_{1}: 70 \%$ y a $: 60 \%$

Factor B: Plantas Medicinales. $\mathrm{b}_{0}$ : Hierba Luisa, $\mathrm{b}_{1}$ : Guayusa, $\mathrm{b}_{2}$ : Menta

\section{Análisis fisicoquímicos}

\section{Determinación de la composición proximal}

La determinación del contenido de humedad se efectuó de acuerdo con el método AOAC 925.10 (AOAC, 2012); el método gravimétrico de AOAC 2003.06 (AOAC, 2012) para la determinación del contenido de lípidos; el método AOAC 2001.11 (AOAC, 2012) para la proteína; el método AOAC 985.29 (AOAC, 2012) para la fibra dietética total y el método AOAC 923.03 (AOAC, 2012) para la ceniza. El contenido de carbohidrato se obtuvo por diferencia.

\section{Análisis de pH, acidez y sólidos solubles}

Se realizó las mediciones de $\mathrm{pH}$ de acuerdo con la norma "INEN-ISO, 1842:2013 Productos vegetales y de frutas. Determinación de pH (IDT)". El grado de acidez fue determinado por un método potenciométrico de acuerdo con la norma "INEN, 381 Conservas vegetales. Determinación de la Acidez Titulable. Método potencio métrico de referencia", expresando como g ácido cítrico (AC) g de muestra ${ }^{-1}$ (P). El contenido de sólidos solubles (SS) fue determinado usando un refractómetro y expresado en ${ }^{\circ}$ brix.

\section{Determinación de polifenoles totales}

Los polifenoles totales fueron determinados según el método colorimétrico de Folin-Ciocalteu (Rodríguez et al., 2014), usando un extracto obtenido con una solución acuosa de metanol al 70\% como solvente, mediante agitación magnética continua por 45 minutos y filtración sobre papel filtro Whatman $\mathrm{N}^{\circ} 1$. Se tomó $0,5 \mathrm{~mL}$ de una parte alícuota del extracto, al cual se agregó $0,5 \mathrm{~mL}$ del reactivo de Folin-Ciocalteu (FC). Luego de 5 min se agregó $2 \mathrm{~mL}$ de una solución de carbonato de sodio (200 mg Na $\mathrm{CO}_{3} \mathrm{~mL}^{-1}$ ) y se mezcló por $15-20 \mathrm{~s}$ usando un agitador vortex. Se deja reaccionar por $15 \mathrm{~min}$ a temperatura ambiente y protegido de la luz solar. Se agregó $10 \mathrm{~mL}$ de agua bidestilada y se elimina el precipitado por centrifugación a 4000x g por $5 \mathrm{~min}$. Se midió la absorbancia a $760 \mathrm{~nm}$ con un espectrofotómetro (Raleigh, UV-1601, China) y determinó la concentración usando una curva de calibración establecida con diferentes concentraciones de ácido gálico. Se realizó la medición en triplicado y se expresó la concentración en mg de ácido gálico equivalente (AGE) por $100 \mathrm{~g}$ de muestra. 


\section{Determinación de capacidad antioxidante ORAC y DPPH}

Para la determinación de la capacidad antioxidante por el ensayo ORAC (Oxygen Radical Absorbance Capacity) se procedió según el método descrito por Ou et al. (2002) con leve modificación. Se preparó el extracto con 1000,0 $\pm 0,1 \mathrm{mg}$ de la cascarilla de cacao y $20 \mathrm{~mL}$ de acetona acuosa $(50: 50 \mathrm{v} / \mathrm{v})$, macerando por $1 \mathrm{~h} \mathrm{a}$ $200 \mathrm{rpm}$ sobre un agitador orbital. El macerado fue centrifugado por $15 \mathrm{~min}$ a $4000 \mathrm{~g}$ y el sobrenadante fue filtrado sobre un filtro Whatman $\mathrm{N}^{\circ} 1$. El proceso de extracción por maceración fue repetido y la mezcla de sobrenadantes evaporada a $40^{\circ} \mathrm{C}$ usando un rotavapor con vacío. Se agregó al residuo del rotavapor $25 \mathrm{~mL}$ de buffer fosfato al $75 \mathrm{mM}(\mathrm{pH} 7,0 \pm 0,2)$ para obtener el extracto usado en el análisis. El ensayo ORAC fue realizado con el antioxidante Trolox como referencia y en un espectrofluorómetro de microplacas de doble exploración (Spectra MAX Gemini, USA). Para la medición, se preparó la solución de fluoresceína al $100 \mathrm{mM}$ con $75 \mathrm{mM}$ de buffer fosfato (pH 7,4), la cual se mantuvo en ambiente oscuro. En cada pocillo de la microplaca se mezcló $45 \mu \mathrm{L}$ extracto con $175 \mu \mathrm{L}$ fluoresceína $(70 \mathrm{nM}$ concentración final) y se incubó por $30 \mathrm{~min}$ a $37^{\circ} \mathrm{C}$, para agregar prontamente $50 \mu \mathrm{L}$ AAPH $\left(2,2^{\prime}\right.$-azobis-2-amidinopropano-dihidrocloruro) en solución (20 mM concentración final). Se midió la fluorescencia a temperatura constante de $37^{\circ} \mathrm{C}$ por alrededor de $60 \mathrm{~min}$, registrando la lectura cada 3 min hasta una lectura de $<5 \%$ del valor inicial. En paralelo, se realizó un testigo (con buffer fosfato sin extracto) y 4 soluciones de Trolox a concentraciones de 6, 12, 18 y $24 \mathrm{mM}$. Los valores ORAC se expresaron como mM equivalente Trolox (ET) por $100 \mathrm{~g}$ muestra.

La capacidad antioxidante como valor DPPH fue determinada por el método de decoloración del radical libre 2,2-Difenil-l-picrilhidrazilo (DPPH) según BrandWilliams et al. (1995). Al extracto se agregó 2,9 mL de DPPH en metanol con una absorbancia inicial de 0,8 medida a $515 \mathrm{~nm}$. Después de 30 min se lee la absorbancia final, lo que se compara con valores de inhibición de una curva de calibración preparada con el reactivo de Trolox a concentraciones de 250, 500, 750 y $1000 \mathrm{mM}^{2}$ Trolox mL $\mathrm{m}^{-1}$. Los valores DPPH se expresaron como mM ET 100 g muestra $^{-1}$.

\section{Evaluación sensorial}

Se efectuó el análisis sensorial de la infusión con un diseño factorial de bloques incompletos, evaluando los tratamientos mediante los atributos sensoriales: color, aroma, sabor y aceptabilidad. Los análisis fueron realizados con estudiantes que cursaban los últimos semestres de la carrera de Ingeniería en Alimentos de la Facultad de Ciencia e Ingeniería en Alimentos, de la
Universidad Técnica de Ambato, en Ecuador, que actuaban como jueces semi-entrenados. Hubo un total de 18 jueces semi-entrenados y cada catador analizó cinco muestras al azar, de forma que existieron nueve réplicas para cada tratamiento. Se utilizó una escala hedónica estructurada de 5 puntos, siendo " 1 " "desagrada mucho" y "5" "agrada mucho". Los datos fueron analizados por el programa estadístico Statgraphics Centurion XV.

\section{Análisis microbiológico}

El análisis microbiológico fue realizado en triplicado sólo como control al mejor tratamiento. Para el conteo microbiológico de mohos y levaduras, aerobios mesófilos, Escherichia coli y Staphylococcus aureus, se utilizó las siguientes normas:

- INEN, 1529-5 Control microbiológico de los alimentos. Determinación de la cantidad de microorganismos aerobios mesófilos.

- INEN, 1529-11:98 Control microbiológico de alimentos mohos y levaduras viables detección.

- INEN, 1529-7:2013 Control Microbiológico de los alimentos. Determinación de microorganismos coliformes por la técnica de recuento de colonias.

- INEN, 1529-14:2013 Control microbiológico de los alimentos Staphylococcus aureus. Recuento en placa de siembra por extensión en superficie.

\section{Determinación del tiempo de vida útil}

Para la determinación de la vida útil se empleó un test acelerado de tiempo a tres diferentes temperaturas $\left(18,25,35^{\circ} \mathrm{C}\right)$ durante 96 días. Se utilizó la aproximación de Arrhenius.

$$
\operatorname{Ln} \mu=\operatorname{Ln} A \frac{E_{\mathrm{a}}}{(R * T)}
$$

Donde:

$\mu \quad$ Velocidad especifica de crecimiento (ufc $\mathrm{g}^{-1} \mathrm{~h}^{-1}$ )

Ln $A$ Intercepto de la recta Ln $\mu$ vs $1 / T$

$A \quad$ Constante (ufc g ${ }^{-1} \mathrm{~h}^{-1}$ )

$E_{\text {a }} \quad$ Energía de activación $\left(\mathrm{J} \mathrm{kmol}^{-1}\right)$

$R \quad$ Constante universal de los gases $\left(8314 \mathrm{~J} \mathrm{kmol} \mathrm{K}^{-1}\right)$

$T$ Temperatura en $\mathrm{K}$

Se utilizó como referencia para el recuento máximo permitido el valor de $10^{4} \mathrm{ufc} \mathrm{g}^{-1}$ estipulado en la "Norma Técnica Ecuatoriana (INEN, 2392-2013) Hierbas Aromáticas. Requisitos". 


\section{RESULTADOS}

\section{Análisis fisicoquímicos}

Contenido de lípidos, proteína, cenizas, fibra dietética y carbohidratos

El contenido de lípidos determinado en la cascarilla de cacao variedad Arriba fue de 5,37 $\pm 0,08 \%$, lo que se encuentra dentro del rango entre 1 al $6 \%$ reportado por Lecumberri et al. (2007). En las muestras de hierba luisa, guayusa y menta el contenido de lípidos estuvo por debajo del límite de detección, por lo que no aporta este componente a la infusión. El contenido de proteína $(15,66 \%)$, ceniza $(8,41 \%)$, fibra dietética total $(6,26 \%)$ y carbohidratos $(59,89 \%)$ fueron determinados sólo para la cascarilla de cacao variedad Arriba.

\section{Contenido de humedad}

En la cascarilla de cacao variedad Arriba el contenido de humedad promedio de tres réplicas fue de 9,78 $\pm 0,01 \%$, mientras que en la muestra de hierba luisa fue de $9,55 \pm 0,15 \%$, en la guayusa $10,03 \pm 0,14 \%$ y en la menta $9,85 \pm 0,08 \%$. Esto coincide con los valores entre 5,4 y $15,3 \%$ reportado por EFSA (2008). Para la preparación de la infusión con diferentes proporciones de cascarilla de cacao con las diferentes hierbas el contenido de humedad fluctuó entre 8,25 \pm 0,00\% en la mezcla de $70 \%$ de cascarilla con hierba luisa $\left(a_{1} b_{0}\right)$ y $9,91 \pm 0,00 \%$ en la mezcla de $80 \%$ de cascarilla con hierba luisa $\left(\mathrm{a}_{0} \mathrm{~b}_{0}\right)$, tal como se muestra en el Cuadro 1. Se observó que el contenido de humedad en las mez- clas para la infusión fue diferente a lo calculado, lo que se puede considerar como una fluctuación en productos naturales. El análisis de varianza muestra los factores: \% de cascarilla (Factor $\mathrm{A}$ ) y hierbas aromáticas (Factor B) y, demostró diferencias no significativas $(\mathrm{p}<0,05)$ sobre la respuesta experimental en \% de humedad, al igual que el efecto combinado de los mismos en los tratamientos.

\section{El pH en la infusión}

Se realizó mediciones en triplicado de $\mathrm{pH}$ para cada tratamiento y el promedio de las determinaciones se muestra en la Cuadro 1. Este parámetro permite controlar el proceso de descomposición de la infusión debido a que los microorganismos sólo pueden crecer en un rango estrecho de $\mathrm{pH}$. El análisis de varianza de las medidas de $\mathrm{pH}$, demuestra que existen diferencias significativas $(\mathrm{p}<0,05)$ en $\%$ de cascarilla (Factor $A$ ) y hierbas aromáticas (Factor B), lo que influyen significativamente sobre la respuesta experimental al igual que el efecto combinado de los mismos.

\section{Análisis de acidez en la infusión}

El grado de acidez de las distintas infusiones se expresó como acidez titulable en g de ácido cítrico por gramo de producto ( $\mathrm{g} \mathrm{AC} \mathrm{g} \mathrm{P}^{-1}$ ). Los resultados se muestran en el Cuadro 1. El análisis de varianza de las medidas de acidez arrojó que no existe diferencias significativas ( $p>0,05)$ en el grado de acidez entre las infusiones con diferentes \% de cascarilla (Factor A) y hierbas aromáticas (Factor B), lo que significa que estos facto-

Cuadro 1. Características fisicoquímicas de las mezclas para infusión.

Table 1. Physicochemical characteristics of the infusion blends.

\begin{tabular}{|c|c|c|c|c|}
\hline \multirow[t]{2}{*}{ Tratamientos } & Humedad & $\mathrm{pH}$ & Acidez & SS \\
\hline & $\%$ & & $\mathrm{~g} \mathrm{AC} \mathrm{g} \mathrm{P}^{-1}$ & obrix \\
\hline$a_{0} b_{0}$ & $9,91 \pm 0,00^{\mathrm{a}}$ & $6,93 \pm 0,06^{b}$ & $0,01+0,00^{a}$ & $2,00+0,00^{\mathrm{a}}$ \\
\hline$a_{0} b_{1}$ & $9,42 \pm 0,1^{8} \mathrm{c}$ & $6,64 \pm 0,04^{\mathrm{ab}}$ & $0,01+0,00^{\mathrm{a}}$ & $4,00+0,00^{\mathrm{bc}}$ \\
\hline$a_{0} b_{2}$ & $8,95 \pm 0,00^{\mathrm{d}}$ & $7,20 \pm 0,01^{\mathrm{a}}$ & $0,01+0,00^{\mathrm{a}}$ & $3,00+0,00^{\mathrm{ab}}$ \\
\hline$a_{1} b_{0}$ & $8,25 \pm 0,00^{\mathrm{e}}$ & $7,20 \pm 0,02^{\mathrm{a}}$ & $0,01+0,00^{a}$ & $2,00+0,00^{\mathrm{a}}$ \\
\hline$a_{1} b_{1}$ & $8,92 \pm 0,00^{d}$ & $6,59 \pm 0,09^{a b}$ & $0,01+0,00^{a}$ & $3,00+0,00^{\mathrm{ab}}$ \\
\hline$a_{1} b_{2}$ & $9,65 \pm 0,00^{b}$ & $6,91 \pm 0,13^{\mathrm{b}}$ & $0,01+0,00^{\mathrm{a}}$ & $2,00+0,00^{\mathrm{a}}$ \\
\hline$a_{2} b_{0}$ & $8,95 \pm 0,00^{\mathrm{d}}$ & $7,27 \pm 0,06^{\mathrm{a}}$ & $0,01+0,00^{\mathrm{a}}$ & $2,00+0,00^{\mathrm{a}}$ \\
\hline$a_{2} b_{1}$ & $9,34 \pm 0,00^{c}$ & $6,83 \pm 0,06^{b}$ & $0,02+0,00^{\mathrm{a}}$ & $4,00+0,00^{b c}$ \\
\hline$a_{2} b_{2}$ & $8,93 \pm 0,00^{\mathrm{d}}$ & $7,28 \pm 0,00^{a}$ & $0,01+0,00^{\mathrm{a}}$ & $2,00+0,00^{\mathrm{a}}$ \\
\hline Testigo & $9,63 \pm 0,01^{b}$ & $6,85 \pm 0,05^{b}$ & $0,01+0,00^{\mathrm{a}}$ & $2,00+0,00^{\mathrm{a}}$ \\
\hline
\end{tabular}

Letras distintas en la misma columna indican diferencias significativas $(\mathrm{p}<0,05)$. 
res no influyen sobre la respuesta experimental al igual que el efecto combinado de los mismos.

\section{Sólidos solubles en la infusión}

Los valores de sólidos solubles determinados en las distintas infusiones fluctuaron entre 2 y $4{ }^{\circ} \mathrm{brix}$, tal como se presenta en el Cuadro 1. El análisis de varianza de sólidos solubles en ${ }^{\circ}$ brix arrojó leves pero significativas diferencias $(p>0,05)$ entre las distintas infusiones con distintos \% de cascarilla (Factor A) y hierbas aromáticas (Factor B), lo cual se puede atribuir a distintas cantidades de sólidos solubles que contienen cada una de las plantas deshidratadas.

\section{Análisis de polifenoles totales y capacidad antioxi- dante}

En la muestra desengrasada de cascarilla de cacao se determinó un contenido de polifenoles totales de

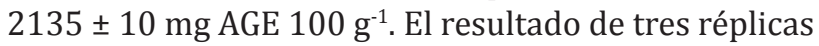
del análisis de polifenoles totales en las muestras de las hierbas aromáticas fue de un promedio de $468 \pm 10$; $662 \pm 10$ y $977 \pm 10 \mathrm{mg} \mathrm{AGE} \mathrm{g}^{-1}$ para la hierbaluisa, la guayusa y la menta, respectivamente. En una muestra comparativa de cascarilla de cacao adquirido en una tienda de productos naturales de la ciudad de Valdivia se determinó un menor contenido de polifenoles totales de $646 \pm 41 \mathrm{mg}$ AGE $100 \mathrm{~g}^{-1}$ con una capacidad antioxidante en ORAC y DPPH de $10656 \pm 473$ y $1732 \pm$ 55 mmol Trolox $100 \mathrm{~g}^{-1}$ de muestra, respectivamente, lo que indicó pérdidas de hasta $70 \%$ comparadas con un producto de origen.

\section{Evaluación sensorial}

El ensayo se realizó con 18 jueces semi-entrenados y el análisis estadístico de la evaluación sensorial de la infusión estableció que el tratamiento $\mathrm{a}_{0} \mathrm{~b}_{1}(80 \%$ cascarilla de cacao - $20 \%$ guayusa) fue el mejor obteniendo la mayor valoración por los catadores en todos los atributos tanto de color como de sabor y aceptabilidad. El tratamiento $\mathrm{a}_{1} \mathrm{~b}_{0}(70 \%$ cascarilla de cacao - $30 \%$ guayusa) fue encontrado como el segundo mejor tratamiento por su alta valoración en aceptabilidad y sabor, pero con tercer lugar en aceptación del olor. El tratamiento $a_{1} b_{0}(70 \%$ cascarilla de cacao - $30 \%$ hierba luisa) fue el tercer mejor tratamiento por ser segundo con mayor valoración en color, sabor y tercero en olor y aceptabilidad.

\section{Análisis microbiológico}

El análisis microbiológico de alimentos no tiene carácter preventivo, sino simplemente es una inspección que permite valorar la carga microbiana. Para el mejor tratamiento se determinó el recuento de mohos y levaduras, aerobios mesófilos, Escherichia coli y Staphylococcus aureus, basándose en la norma INEN 2392, Ecuador. Estas pruebas se realizaron al haber concluido la elaboración de la infusión, que fue almacenada a las temperaturas de $18 \pm 2{ }^{\circ} \mathrm{C} ; 25 \pm 2{ }^{\circ} \mathrm{C}$ y $35 \pm 2{ }^{\circ} \mathrm{C}$. Los resultados microbiológicos obtenidos cumplieron con lo especificado en las normas establecidas, por lo que el producto es seguro en los aspectos higiénicos.

\section{Test de vida útil}

Se hizo una evaluación de la estabilidad microbiológica de la mejor combinación de la cascarilla de cacao con la hierba aromática de guayusa, $\mathrm{a}_{0} \mathrm{~b}_{1}(80 \%$ cascarilla de cacao $-20 \%$ guayusa), utilizando el recuento de mohos y levaduras como indicador y la ecuación de Arrhenius para modelar el desarrollo de los microorganismos indicadores. Los resultados de la cinética de crecimiento a tres temperaturas $\left(18,25\right.$ y $\left.35^{\circ} \mathrm{C}\right)$ se muestran en la Figura 1, mientras que en la Figura 2 se visualiza el cálculo de la vida útil de acuerdo con los criterios según la Norma INEN 2392, Ecuador. El tiempo de vida útil de la infusión estimado mediante el test acelerado de tiempo a las tres temperaturas durante 96 días arrojó un tiempo óptimo de 147,6 días a $18{ }^{\circ} \mathrm{C}$. A una temperatura más elevada de $35{ }^{\circ} \mathrm{C}$ la vida útil se reduce a casi 63 días.

\section{CONCLUSIONES}

La caracterización fisicoquímica realizada con el fin de determinar las propiedades de la cascarilla de cacao variedad Arriba, sola o mezclada con hierbas aromáticas demostró que las infusiones obtenidas son levemente ácidas con mayor tendencia al neutro y alcanzaron una concentración entre 2 y 4 ºbrix, lo que le otorgó una buena aceptabilidad por los degustadores. Las infusiones contienen un alto contenido de antioxidantes determinados como polifenoles totales que proviene principalmente de la cascarilla de cacao con $2135 \mathrm{mg}$ AGE g-1 . La combinación de $80 \%$ de cascarilla de cacao con $20 \%$ de guayusa tuvo la mayor aceptabilidad, indicando preferencia por una infusión levemente ácido con pH 6,64 y un contenido de sólidos solubles de 4 ${ }^{\circ}$ brix. Esta combinación de cascarilla de cacao con guayusa es microbiológicamente segura y estable a $18{ }^{\circ} \mathrm{C}$ con una vida útil de casi 148 días.

\section{AGRADECIMIENTOS}

Esta obra fue financiada por la Universidad Técnica de Ambato en el marco del Proyecto Semilla "Mejoramiento del Proceso de Fermentación del Cacao (Theobroma cacao L.) Variedad Nacional y Variedad CCN51" 


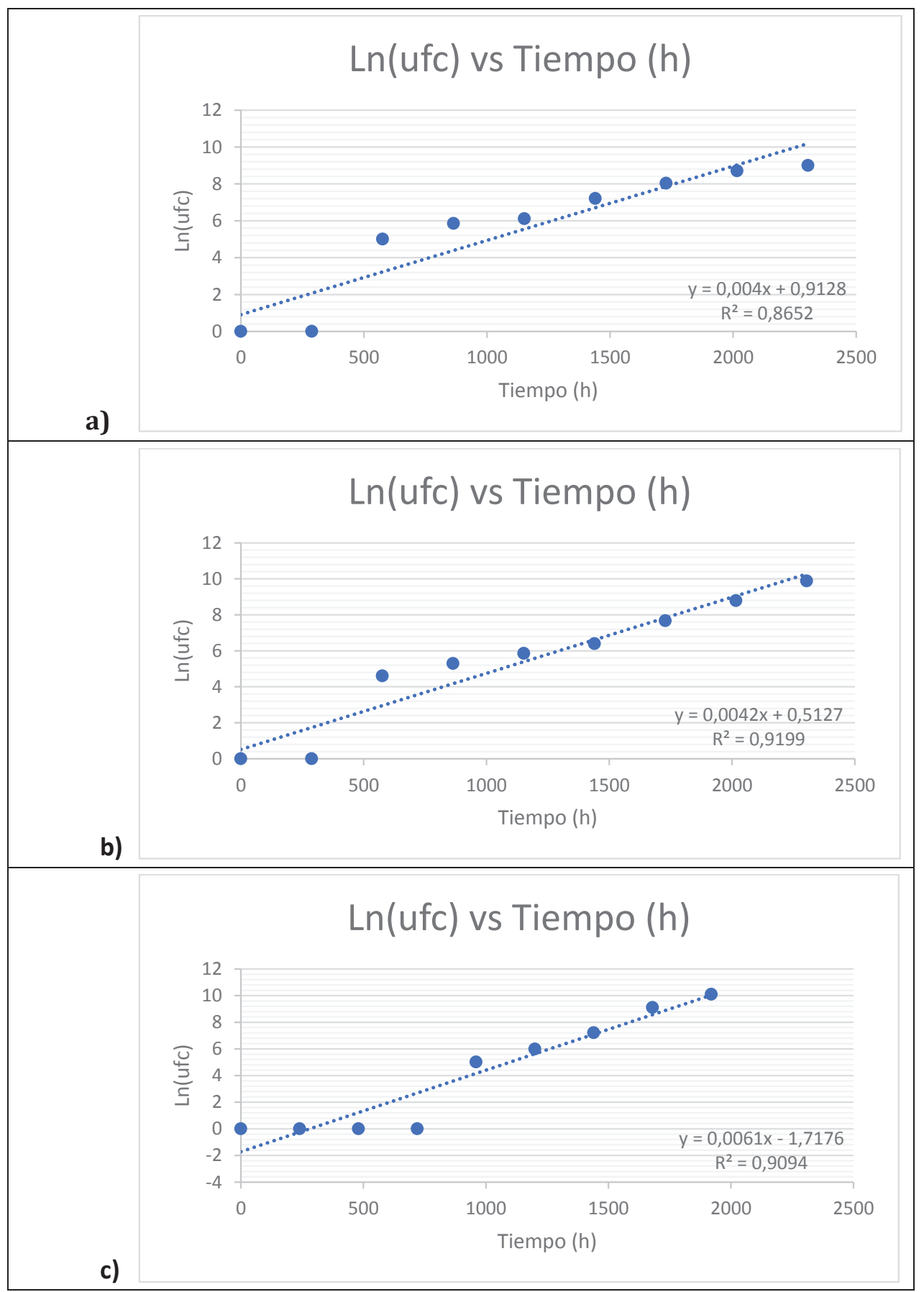

Figura 1. Test acelerado de vida útil para la mezcla de infusión de $\mathrm{a}_{0} \mathrm{~b}_{1}$ (cascarilla $80 \%$ y guayusa 20\%). Temperatura de ensayos a) $18^{\circ} \mathrm{C}$; b) $25^{\circ} \mathrm{C}$ y c) $35^{\circ} \mathrm{C}$.

Figure 1. Accelerated shelf-life test for the infusion blend $\mathrm{a}_{0} \mathrm{~b}_{1}\left(80 \%\right.$ husks and $20 \%$ guayusa). Test temperature a) $18{ }^{\circ} \mathrm{C}$; b) $25^{\circ} \mathrm{C} \mathrm{y} \mathrm{c)} 35^{\circ} \mathrm{C}$. 


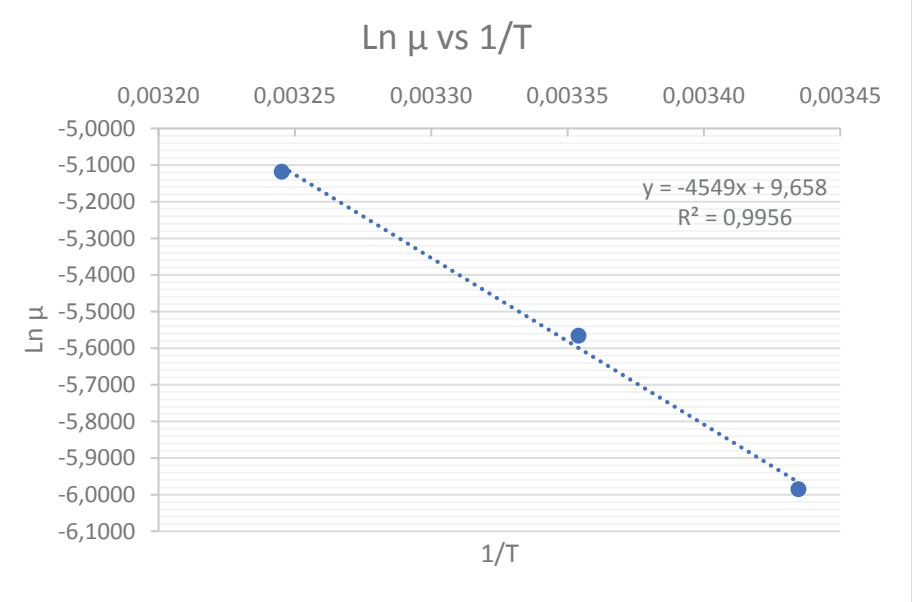

\begin{tabular}{|c|c|c|c|c|c|}
\hline Tratamiento & $\mathbf{T}$ & ${ }^{\circ} \mathbf{C}$ & $\mathbf{1 8}$ & $\mathbf{2 5}$ & $\mathbf{3 5}$ \\
\hline \multirow{4}{*}{$\mathrm{a}_{0} \mathrm{~b}_{1}$} & ${ }^{1} m$ & $\mathrm{~K}$ & \multicolumn{3}{|c|}{$-4548,995$} \\
\cline { 2 - 6 } & ${ }^{2} \mathrm{Ln} A$ & - & \multicolumn{3}{|c|}{9,65801} \\
\cline { 2 - 6 } & ${ }^{3} E_{\mathrm{a}}=-(m R)$ & $\mathrm{J} / \mathrm{mol}$ & \multicolumn{3}{|c|}{37822,17} \\
\cline { 2 - 6 } & ${ }^{4} \mu$ & $\mathrm{h}^{-1}$ & 0,0026 & 0,0037 & 0,0061 \\
\cline { 2 - 6 } & ${ }^{5} \mathrm{Ln} R M_{\text {máx }}$ & - & \multicolumn{3}{|c|}{9,2103404} \\
\cline { 2 - 6 } & ${ }^{6} T V U$ & días & 147,6 & 103,7 & 62,9 \\
\hline
\end{tabular}

${ }^{1}$ Pendiente de la recta $\operatorname{Ln} \mu$ versus $1 / T$

${ }^{2}$ Intercepto de la recta $\operatorname{Ln} \mu$ versus $1 / T$

${ }^{3}$ Energia de activación dependiente de la pendiente $m$ y la constante de gas ideal $R$

${ }^{4}$ Valor de $\mu$ calculado a partir de la ecuación $\operatorname{Ln} \mu=\operatorname{Ln} A-\left(E_{\mathrm{a}} / R T\right)$

${ }^{5} \mathrm{Ln}$ del recuento microbiológico máximo $R M_{\text {máx }}$ en ( $\mu \mathrm{fc} \mathrm{g}^{-1}$ ) según Norma INEN 2392

${ }^{6}$ El tiempo de vida útil se determina mediante $T V U=\ln R M_{\text {máx }} / \mu$

Figura 2. Cálculo de vida útil para la mezcla de infusión de $\mathrm{a}_{0} \mathrm{~b}_{1}$ (cascarilla $80 \%$ y guayusa 20\%).

Figure 2. Determination of shelf-life for the infusion blend $a_{0} b_{1}(80 \%$ husks and $20 \%$ guayusa).

\section{REFERENCIAS}

Arlorio, M., Coïsson, J.D., Travaglia, F., Varsaldi, F., Miglio, G., Lombardi, G., Martelli, A., 2005. Antioxidant and biological activity of phenolic pigments from Theobroma cacao hulls extracted with supercritical $\mathrm{CO}_{2}$. Food Research International 38(8-9), 1009-1014. https://doi. org/10.1016/j.foodres.2005.03.012

Association of Official Analytical Chemists (AOAC), 2012. Official methods of analysis of AOAC International, $16^{\text {th }} \mathrm{ed}$., AOAC International. Maryland, Estados Unidos.

Blanco, M.M., Costa, C.A., Freire, A.O., Santos, J.G., Costa, M., 2009. Neurobehavioral effect of essential oil of Cymbopogon citratus in mice. Phytomedicine 16(2-3), 265270. https://doi.org/10.1016/j.phymed.2007.04.007

Brand-Williams, W., Cuvelier, M.E., Berset, C., 1995. Use of a free radical method to evaluate antioxidant activity. LWT-Food Science and Technology 28(1), 25-30. https://doi.org/10.1016/S0023-6438(95)80008-5

Castillejo, G., Millán, S., Bulló, M., Anguera, A., Escribano, J., Salas-Salvadó, J., 2006. Estudio controlado, randomizado, a doble estudio ciego, evaluando el efecto de un suplemento de cáscara de cacao rico en fibra sobre el tránsito colónico en pacientes pediátricos con constipación. Nutrición Hospitalaria 21(1), 91.

Correa, J., Bernal, H., 1989. Especies vegetales promisorias de los países del Convenio Andrés Bello. Programa de Recursos Vegetales del Convenio Andrés Bello (PREVACAB), Bogotá, Colombia.

Dávila, J., Calero, A., Roldán, S., Benítez, F., 2010. Ingredientes funcionales de plantas ecuatorianas. Revista Politécnica 29(1), 51-59. 
De la Mota, I.H., 2007. El libro del chocolate. Editorial Pirámide, Madrid, España.

De la Torre, L., Navarrete, H., Muriel, P., Macía, M.J., Balslev, H., 2008. Enciclopedia de las plantas útiles del Ecuador. Herbario QCA \& Herbario AAU, Quito y Aarhus.

Desam, N.R., Al-Rajab, A.J., Sharma, M., Mylabathula, M.M., Gowkanapalli, R.R., Albratty, M., 2017. Chemical constituents, in vitro antibacterial and antifungal activity of Mentha x Piperita L. (peppermint) essential oils. Journal of King Saud University - Science «in press» http:// dx.doi.org/10.1016/j.jksus.2017.07.013

Dueñas, J.F., Jarrett, C., Cummins, I., Logan-Hines, E., 2016. Amazonian Guayusa (Ilex guayusa Loes.): a historical and ethnobotanical overview. Economic Botany 70(1), 85-91. https://doi.org/10.1007/s12231-016-9334-2

European Food Safety Authority (EFSA), 2008. Theobromine as undesirable substances in animal feed - Scientific opinion of the Panel on Contaminants in the Food Chain. The EFSA Journal 725, 1-66. https://doi.org/10.2903/j. efsa.2008.725

Figueirinha, A., Cruz, M.T., Vera, F., Lopes, M.C., Batista, M.T., 2010. Anti-inflammatory activity of Cymbopogon citratus leaf infusion in lipopolysaccharide-stimulated dendritic cells: contribution of the polyphenols. Journal of Medicinal Food 13(3), 681-690. https://doi. org/10.1089/jmf.2009.0115

Higdon, J.V., Frei, B., 2003. Tea catechins and polyphenols: health effects, metabolism, and antioxidant functions. Journal Critical Reviews in Food Science and Nutrition. 43(1), 89-143. https://doi. org/10.1080/10408690390826464

Holland, B., Welch, A.A., Unwin, J.D., Buss, D.H., Paul, A.A., 1991. McCance and Widdowson's the composition of foods, $5^{\text {th }}$ ed., Royal Society of Chemistry, London.

Instituto Nacional de Investigaciones Agropecuarias (INIAP), 1994. Manual del cultivo de cacao, $2^{\mathrm{a}}$ ed. Manual No. 25, INIAP-PROTECA, Quevedo, Ecuador, pp. 5-23. https:// es.scribd.com/document/336757996/Manual-N25-MANUAL-DEL-CULTIVO-DE-CACAO

Kapp, K., Hakala, E., Orav, A., Pohjala, L., Vuorela, P., Püssa, T., Vuorela, H., Raal, A., 2013. Commercial peppermint (Mentha $\times$ piperita L.) teas: antichlamydial effect and polyphenolic composition. Food Research International 53(2), 758-766. https://doi.org/10.1016/j.foodres.2013.02.015

Kuriyama, S., 2008. The relation between green tea consumption and cardiovascular disease as evidenced by epidemiological studies. The Journal of Nutrition 138(8), 1548S-1553S. https://doi.org/10.1093/jn/138.8.1548S

Lecumberri, E., Mateos, R., Izquierdo-Pulido, M., Rupérez, P., Goya, L., Bravo, L., 2007. Dietary fibre composition, an- tioxidant capacity and physico-chemical properties of a fibre-rich product from cocoa (Theobroma cacao L.). Food Chemistry 104(3), 948-954.

Lerceteau, E., Flipo, S., Quiroz, J., Soria, J., Pétiard, V., Crouzilat, D., 1997. Genetic differentiation among Ecuadorian Theobroma cacao L. accessions using DNA and morphological analyses. Euphytica 95(1), 77-87. https://doi. org/10.1023/A:1002993415875

López, P., 2013. Elaboración de compost a partir de cascarilla de cacao. Tesis de Grado Bioquímico Farmacéutico. Escuela Superior Politécnica De Chimborazo, Riobamba, Ecuador. 94 p.

Maiti, S., Raju, S., Geetha, K., Mandal, K., 2006. Good agricultural practices for patchouli, geranium and lemongrass. National Research Centre for Medicinal and Aromatic Plants, Boriavi, Gujarat, India.

Marcillo, E.M., Naranjo, D., 2012. Diseño de la línea de producción de una bebida de hierbas denominada horchata. Tesis de Grado Ingeniero en Alimentos, Escuela Superior Politécnica del Litoral, Guayaquil, Ecuador, 11 p.

Murillo, I., 2008. Evaluación de 2 dietas experimentales con diferentes niveles de cascarilla de cacao (Theobroma cacao L.) en las fases de crecimiento y acabado de cuyes (Cavia porcellus L.) de raza andina. Tesis de Grado Ingeniero en Alimentos. Escuela Superior Politécnica del Litoral, Guayaquil, Ecuador. 145 p.

Nestlé, 2017. El cultivo de cacao en Nestlé. https://empresa. nestle.es/es/cvc/desarrollo-rural/cultivo-cacao (acceso, 13.11.2017).

Ou, B.X., Huang, D.J., Hampsch-Woodill, M., Flanagan, J.A., Deemer, E.K., 2002. Analysis of antioxidant activities of common vegetables employing oxygen radical absorbance capacity (ORAC) and ferric reducing antioxidant power (FRAP) assays: a comparative study. Journal of Agricultural and Food Chemistry 50(11), 3122-3128. https:// doi.org/10.1021/jf0116606

Perego, P., Fabiano, B., Cavicchioli, M., Del Borghi, M., 2004. Cocoa quality and processing: a study by solid-phase microextraction and gas chromatography analysis of methylpyrazines. Food and Bioproducts Processing 82(4), 291-297. https://doi.org/10.1205/ fbio.82.4.291.56402

Quer, P., 1964. Plantas Medicinales. Labor S.A., Barcelona, España.

Rodríguez, K., Ah-Hen, K., Vega-Gálvez, A., López, J., QuispeFuentes, I., Lemus-Mondaca, R., Gálvez-Ranilla, L., 2014. Changes in bioactive compounds and antioxidant activity during convective drying of murta (Ugni molinae T.) berries. International Journal of Food Science \& Technology 49(4), 990-1000. https://doi.org/10.1111/ ijfs. 12392 
